SELECCIONES MATEMÁTICAS
Universidad Nacional de Trujillo
ISSN: $2411-1783$ (Online)
2019; Vol.06(2): $248-263$.

\title{
Modelo de optimización con programación no lineal y simulación Monte Carlo de un proyecto industrial pesquero
}

\section{Optimization model with nonlinear programming and Monte Carlo simulation of an industrial fishing project}

\author{
Christian Ramos Angeles*(iD) and Pedro Espinoza Haro.
}

Received, Jul. 20, 2019

Accepted, Set. 06, 2019

How to cite this article:

Ramos C., Espinoza P. Modelo de optimización con programación no lineal y simulación Monte Carlo de un proyecto industrial pesquero. Selecciones Matemáticas. 2019; 6(2):248-263. http://dx.doi.org/10.17268/sel. mat. 2019.02.11

\section{Resumen}

El presente trabajo de investigación consiste en el desarrollo de un método en donde se plantea un modelo matemático de un proyecto de instalación de una planta de conservas de pescado, considerando la inversión, los costos de producción, los gastos operativos, los estados financieros, y como indicadores para la toma de decisiones el valor actual neto financiero y la tasa interna de retorno financiero. Posteriormente se optimiza el modelo matemático utilizando programación no lineal para elegir la alternativa de inversión más conveniente, luego se realiza una simulación Monte Carlo en donde se consideran variables aleatorias de planta y de mercado que permita al inversionista estimar los valores mínimos y máximos que podrá ocurrir en los indicadores financieros y por último la discusión de resultados.

Palabras claves. Optimización, programación no lineal, simulación Monte Carlo, proyecto industrial.

\begin{abstract}
The present research work consists in the development of a method where a mathematical model of a fish canning plant installation project is proposed, considering the investment, the production costs, the operating expenses, the financial statements, and as indicators for decision making, the net financial present value and internal rate of financial return. Then, the mathematical model is optimized using a non-linear programming to choose the most convenient investment alternative, then a Monte Carlo simulation is carried out in which random variables of plant and market are considered that allow the investor to estimate the minimum values and máximum that can happen in the financial indicators and finally the discussion of results.
\end{abstract}

Keywords. Optimization, nonlinear programming, Monte Carlo simulation, industrial project.

1. Introducción. El inversionista es un tomador de decisiones que siempre busca escoger la mejor alternativa de inversión que maximice las ganancias, considerando que siempre está bajo un entorno cambiante, lo cual hace que sus expectativas planeadas no siempre son las que tendrán.

Si la toma de decisiones es sobre un proyecto industrial pesquero, se tendrá que tener en cuenta que existen muchas variables de decisión como variables aleatorias que se deben de considerar para poder evaluar dicho

\footnotetext{
*Facultad de Pesquería, Universidad Nacional Agraria La Molina, Av. La Universidad S/N, Lima-Perú. (cramoselamolina.edu.pe).

${ }^{\dagger}$ Facultad de Ingeniería Industrial y de Sistemas, Universidad Nacional de Ingeniería, Av. Túpac Amaru 210, Lima-Perú. (pcesp67@gmail.com).
} 
proyecto.

La propuesta del presente trabajo de investigación es de maximizar el valor actual neto financiero de un proyecto industrial pesquero y realizar simulaciones con el método Monte Carlo.

Existen muchos antecedentes sobre modelos de optimización y simulación aplicados en los proyectos, aplicando programación matemática en la decisión óptima de proyectos en un mercado de capital imperfecto [16] o un modelo matemático dimensional para la planeación de proyectos industriales [17]. En la selección de proyectos de investigación y desarrollo (I+D) se utilizó un modelo de programación meta entero no lineal [19]. En China desarrollaron un sistema con el uso de modelos y algoritmos de la investigación de operaciones para la evaluación de proyectos del Centro de Información Estatal (SIC) para que administre las inversiones en el Sistema de Información Económica del Estado de China (SEIS) [20]. La programación multiobjetivos difuso también se utilizó en la toma de decisiones en la selección de proyectos I+D [3]. El método de programación de red también fue utilizado en el problema de la cartera óptima de proyectos de inversión [21]. En proyectos de construcción, se desarrollaron modelos de programación lineal para la aplicación en la toma de decisiones en la optimización de la estrategia de licitación para seleccionar la mejor oferta resolviendo problemas de costo y tiempo mediante el uso del software LINDO [1].

En la administración de proyectos se minimizó el costo mediante el uso de modelos de programación no lineal (PNL) [7] y también reduciendo el tiempo de finalización del proyecto con costos no lineales y aproximándolo a un modelo de programación lineal de enteros mixtos (PLEM) [12].

En los portafolios de inversiones se propuso un modelo de programación meta lineal estocástico para la gestión de la cartera de inversiones, considerando como objetivos a la inversión, el control del riesgo, la generación de escenarios [6] y el uso del valor presente neto (VPN) como método cuantitativo tradicional y otro cualitativo como el análisis multicriterio también conocido como el de criterios de inversión de capital no tradicional, integrándolas con técnicas de optimización [5]. También utilizaron modelos de programación lineal multiobjetivo difusa para la optimización de la cartera de inversiones [15].

La aplicación de la simulación en los proyectos de inversión se debe a que en la formulación del proyecto se asumen valores estimados que normalmente cambian durante el ciclo de vida del proyecto [2] e implican riesgo [9] y hay que suponer correctamente las distribuciones de probabilidad de las variables para evitar problemas en los resultados de la simulación [13]. En proyectos ambientales se puede utilizar el método de simulación Monte Carlo definiendo las distribuciones de probabilidad en las variables aleatorias [14] y en otros proyectos de inversión a largo plazo con el desarrollo de modelos determinista, análisis de sensibilidad y probabilístico para el flujo de caja descontado [8]. También se buscaron soluciones óptimas a un proyecto de inversión no rentable cuyos costos de liquidación evolucionan estocásticamente [4], a una cartera de proyectos bajo incertidumbre y restricciones presupuestarias [10] y en otro caso la combinación de evaluación de proyectos con el uso del valor actual neto, análisis de sensibilidad y simulación Monte Carlo [11].

2. Métodos. En el presente trabajo de investigación se utilizó el siguiente método.

2.1. Caso de estudio. Se desea instalar una planta elaboradora de conservas de anchoveta peruana (Engraulis ringens), presentada en corte tipo tubo, sin cabeza ni vísceras, en envases de hojalata tinapa en salsa de tomate como líquido de gobierno. El peso neto del producto es de $180 \mathrm{~g}$, de los cuales $120 \mathrm{~g}$ son de anchoveta ( 9 piezas) y $60 \mathrm{~g}$ de líquido de gobierno.

En el Cuadro 2.1 se muestra la composición nutricional del contenido de una lata de conserva de anchoveta en salsa de tomate.

CUADRO 2.1

Información nutricional del contenido de una lata de conservas de anchoveta en salsa de tomate

\begin{tabular}{lr}
\hline Característica & Valor nutricional \\
\hline Humedad: & $66.71 \%$ \\
Proteínas: & $17.77 \%$ \\
Grasas: & $10.71 \%$ \\
Carbohidratos: & $1.61 \%$ \\
Cenizas: & $3.20 \%$ \\
Valor calórico: & $166.56 \mathrm{kcal} / 100 \mathrm{~g}$ \\
\hline
\end{tabular}

Para el sinceramiento de la producción se considera los siguientes periodos de trabajo que se muestra en el Cuadro 2.2.

En el Cuadro 2.3 se detalla las capacidades de producción real en cajas por año y las capacidades de procesamiento de kilogramos de anchoveta por día para cada planta. 
CUADRO 2.2

Periodos de trabajo en las plantas de conservas

\begin{tabular}{lr}
\hline \multicolumn{1}{c}{ Periodo } & Cantidad \\
\hline Horas por día & 8 horas \\
Días por mes & 22 días \\
Meses por año & 10 meses \\
\hline
\end{tabular}

CUADRO 2.3

Capacidades de producción de las alternativas de plantas de conservas

\begin{tabular}{lrrr}
\hline Alternativa & $\begin{array}{c}\text { Tamaño de } \\
\text { planta } \\
\text { (cajas/año) }\end{array}$ & $\begin{array}{c}\text { Capacidad de } \\
\text { producción real } \\
\text { (cajas/año) }\end{array}$ & $\begin{array}{c}\text { Capacidad de } \\
\text { procesamiento (kg de } \\
\text { anchoveta/día) }\end{array}$ \\
\hline Planta 1 & 50,000 & 49,000 & 1,436 \\
Planta 2 & 100,000 & 99,000 & 2,873 \\
Planta 3 & 250,000 & 247,000 & 7,182 \\
\hline
\end{tabular}

Para determinar las características de las principales máquinas que se observa en el Cuadro 2.4 se hicieron los cálculos de ingeniería para determinar el requerimiento de vapor de agua para el cocinador continuo, las marmitas, las autoclaves y la capacidad del caldero.

CUADro 2.4

Características de las principales máquinas

\begin{tabular}{lrrrcr}
\hline Alternativa & $\begin{array}{c}\text { Cocinador continuo } \\
\text { (dimensiones) }\end{array}$ & $\begin{array}{c}\text { Cerradora } \\
\text { de latas }\end{array}$ & $\begin{array}{c}\text { Lavadora } \\
\text { de latas }\end{array}$ & $\begin{array}{c}\text { Número de } \\
\text { autoclaves }\end{array}$ & Caldero \\
\hline Planta 1 & $5 \times 1 \mathrm{~m}$ & $70 \mathrm{lpm}$ Canco 06 & $70 \mathrm{lpm}$ & 1 & 376 BHP \\
Planta 2 & $7 \times 1 \mathrm{~m}$ & $1201 \mathrm{lpm}$ Angelus 29p & $120 \mathrm{lpm}$ & 2 & 424 BHP \\
Planta 3 & $10 \times 1.5 \mathrm{~m}$ & $275 \mathrm{lpm}$ Angelus 40p & $275 \mathrm{lpm}$ & 4 & 616 BHP \\
\hline
\end{tabular}

Los tiempos en horas para cada planta se determinaron en base a la eficiencia de los envasadores, a los números de máquinas y capacidades, y se muestra en el Cuadro 2.5.

Considerando que hay un rendimiento del $99 \%$ en la operación de lavado y de $82 \%$ en la operación de drenado, el rendimiento final de materia prima a producto final es de $81.18 \%$. En el programa de producción anual se consideró el supuesto de que iba a haber un crecimiento anual de producción del $3 \%$, y se muestra en el Cuadro 2.6. Como se puede observar el programa de producción es para cinco años que coincide con el periodo de evaluación del proyecto.

Inversión y financiamiento La inversión varía dependiendo del tamaño de planta tal como se observa en el Cuadro 2.7.

En un escenario normal y considerando que se dispone de $\mathrm{S} / 1,000,000$ de capital social y que los porcentajes del préstamo de la inversión fija y capital de trabajo son de $50 \%$ y $60 \%$ respectivamente, se instalará la planta 2 .

2.2. Modelo matemático del proyecto . Para la construcción del modelo matemático del proyecto, se utilizó como referencia un diagrama de influencia del proyecto considerando las variables a utilizar en ella, tal como se muestra en las Figuras 2.1 y 2.2.

2.2.1. Ventas $(\mathbf{V})$. El modelo de las ventas se observa en (2.1), donde $P$ es el precio en soles por caja en los cinco años de venta del producto, $X_{j}$ es la variable binaria que puede tener un valor de 1 si se construye la planta $j$ y 0 de otra manera, donde $j=1, \ldots, 3$, y $Q_{t j}$ es la cantidad de cajas a producir en el año $t$ en la planta $j$, donde $t=1, . ., 5 ; j=1, . ., 3$.

$$
V=P X_{t} Q_{t j}
$$

La capacidad de la planta se muestra en (2.2), donde $Q_{j}$ es la capacidad de planta $j$ en número de cajas de conservas, donde $j=1, . ., 3, T C p$ es la tasa de crecimiento de producción (en nuestro caso igual a $3 \%$ ) y $t$ es el año, donde $t=1, \ldots, 5$.

$$
Q_{t j}=\frac{Q_{j}}{(1+T C p)^{5-t}}
$$


CUADRO 2.5

Tiempo en horas de las principales operaciones unitarias

\begin{tabular}{lccccc}
\hline Alternativa & $\begin{array}{c}\text { Envasado } \\
(\mathrm{h})\end{array}$ & $\begin{array}{c}\text { Cocinado } \\
(\mathrm{h})\end{array}$ & $\begin{array}{c}\text { Preparación de } \\
\text { salsa }(\mathrm{h})\end{array}$ & $\begin{array}{c}\text { Cerrado de } \\
\text { latas }(\mathrm{h})\end{array}$ & $\begin{array}{c}\text { Esterilizado } \\
(\mathrm{h})\end{array}$ \\
\hline Planta 1 & 1.82 & 2.21 & 1.23 & 2.21 & 4.50 \\
Planta 2 & 2.73 & 3.16 & 2.47 & 3.15 & 4.50 \\
Planta 3 & 3.25 & 3.69 & 6.17 & 3.69 & 4.88 \\
\hline
\end{tabular}

CUADRo 2.6

Programa de producción para las plantas de conservas (en cajas por año)

\begin{tabular}{lrrrrr}
\hline Alternativa & \multicolumn{1}{c}{ Año 1 } & \multicolumn{1}{c}{ Año 2 } & \multicolumn{1}{c}{ Año 3 } & \multicolumn{1}{c}{ Año 4 } & \multicolumn{1}{c}{ Año 5 } \\
\hline Planta 1 & 44,424 & 45,745 & 47,130 & 48,544 & 50,000 \\
Planta 2 & 88,849 & 91,514 & 94,260 & 97,087 & 100,000 \\
Planta 3 & 222,122 & 228,785 & 235,649 & 242,718 & 250,000 \\
\hline
\end{tabular}

2.2.2. Costos de producción (CP). En los costos de producción se considera los costos de materiales directos, el costo de la mano de obra directa y los costos indirectos de fabricación tal como se observa en (2.3), donde $C M D$ es el costo de materiales directos (en soles), $C M O D$ es el costo mano de obra directa (en soles), $C I F$ es el costo indirecto de fabricación (en soles), y $D P$ es la depreciación del área de producción (en soles).

$$
C P=C M D+C M O D+C I F+D P
$$

Costo de materiales directos (CMD) Los costos de los materiales directos incluyen el costo de la materia prima, la pasta de tomate, la sal, el agua para la mezcla, los envases, las cajas de cartón y el costo de las etiquetas tal como se puede ver en (2.4), donde Cmp es el costo de materia prima (en soles), $C p t$ es el costo de la pasta de tomate (en soles), $C s$ es el costo de la sal (en soles), Ca es el costo del agua (en soles), $C e$ es el costo de los envases (en soles), $C c c$ es el costo de cajas de cartón (en soles) y Cet es el costo de las etiquetas (en soles).

$$
C M D=C m p+C p t+C s+C a+C e+C c c+C e t
$$

El costo de la materia prima ( $C m p)$ se define en (2.5), donde CUmp es el costo unitario de la materia prima en soles por tonelada (en el proyecto es de S/ 1,675 por tonelada) y Kmp es la constante de conversión en cajas de conserva por tonelada de materia prima (en el proyecto es de 137 cajas/tonelada).

$$
C m p=\frac{C U m p}{K m p} X_{j} Q_{t j}
$$

El costo de la pasta de tomate (Cpt) se define en (2.6), donde $C U p t$ es el costo unitario de la pasta de tomate (en soles por kilogramo) igual a S/ 0.50 por kg y Kpt es la cantidad de pasta de tomate en kilogramos por caja, en el proyecto es de $1.3 \mathrm{~kg}$ por caja.

$$
C p t=C U p t K p t X_{j} Q_{t j}
$$

El costo de la sal (Cs) se define en (2.7), donde $C U s$ es el costo unitario del saco de sal (S/ 0.52 por kg) y $K s$ es la cantidad de sal en kilogramos para una caja de conservas, en el proyecto es de $0.054 \mathrm{~kg}$ por caja.

$$
C s=\frac{C U s K s}{25} X_{j} Q_{t j}
$$

El costo del agua $(\mathrm{Ca})$ se define en (2.8), donde $C U a$ es el costo unitario del agua en soles por metro cúbico igual a S/ 3.9590 por m3 y Ka es la cantidad de agua en litros en una caja de conservas (1.616 litros por caja).

$$
C a=C U a K a X_{j} Q_{t j}
$$

El costo de los envases ( $\mathrm{Ce}$ ) se define en (2.9), donde $C$ ue es el costo unitario de los envases en soles por caja (S/ 20 por caja).

$$
C e=C u e X_{j} Q_{t j}
$$


CUADRO 2.7

Inversión en las plantas de conserva en soles (S/)

\begin{tabular}{lrrr}
\hline \multicolumn{1}{c}{ Inversión } & Planta 1 & Planta 2 & \multicolumn{1}{c}{ Planta 3 } \\
\hline Terreno & 150,000 & 200,000 & 300,000 \\
Edificio & 350,000 & 480,000 & 600,000 \\
Instalaciones & 40,000 & 50,000 & 60,000 \\
Maquinarias y equipos & 300,000 & 340,000 & 400,000 \\
Equipos de oficina & 10,000 & 15,000 & 20,000 \\
Muebles & 10,000 & 16,000 & 20,000 \\
Intangibles & 90,000 & 90,000 & 90,000 \\
\hline Inversión total & $1,150,000$ & $1,591,000$ & $2,190,000$
\end{tabular}

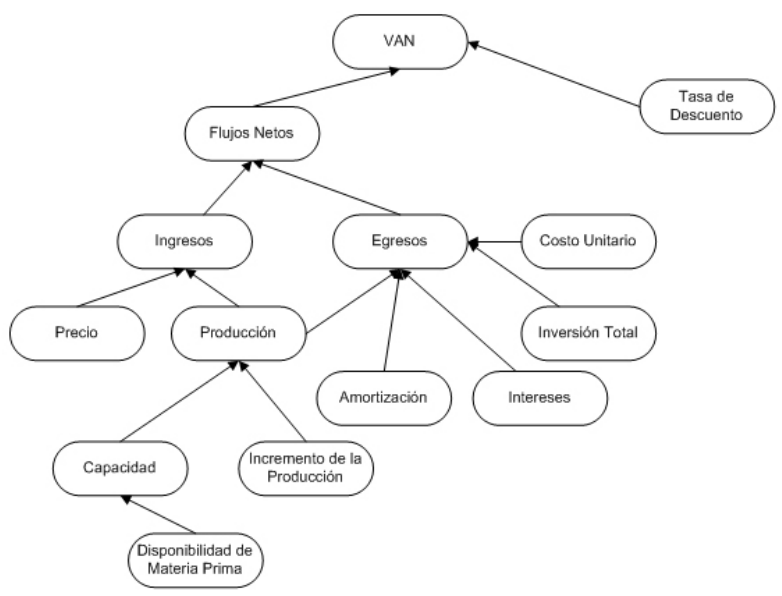

FIGURA 2.1. Diagrama de influencia para el cálculo del VAN

El costo de las cajas de cartón $(C c c)$ se define en (2.10), donde $C U c c$ es el costo unitario de las cajas de cartón en soles por caja (S/ 0.50 por caja).

$$
C c c=C U c c X_{j} Q_{t j}
$$

El costo de las etiquetas (Cet) se define en (2.11), donde CUet es el costo unitario de etiquetas en soles por caja (S/ 0.50 por caja).

$$
C e t=C U \text { et } X_{j} Q_{t j}
$$

Costo de mano de obra directa (CMOD) La mano de obra directa está compuesta por el personal para el proceso, los envasadores, y la alimentación del personal, su costo está definido por (2.12), donde CPp es el costo del personal para proceso, Сep es el costo de envasadores y Cap es el costo alimentación de personal.

$$
C M O D=C p p+C e p+C a p
$$

El costo del personal para proceso ( $C p p$ ) está definido en (2.13), donde $N p p_{j}$ es el número de personal de proceso (depende de la planta, donde $j=1, . ., 3)$, y $C U p p$ es el costo unitario personal para proceso (sueldo de $\mathrm{S} / 750$ por mes).

$$
C p p=N p p_{j}(15 C U p p(1+0,0975)+C U p p) X_{j}
$$

Para el costo del personal de proceso se consideró 15 meses de sueldo (12 sueldos al año, 2 gratificaciones y 1 de vacaciones), así como ESSALUD del $9 \%$ y como es una actividad industrial se paga SENATI equivalente a $0.75 \%$ de los 15 sueldos.

El costo de los envasadores ( $C e p$ ) se muestra en (2.14), donde CUep es el costo unitario de los envasadores al destajo, S/ 1.00 por caja.

$$
C e p=C U e p X_{j} Q_{t j}
$$




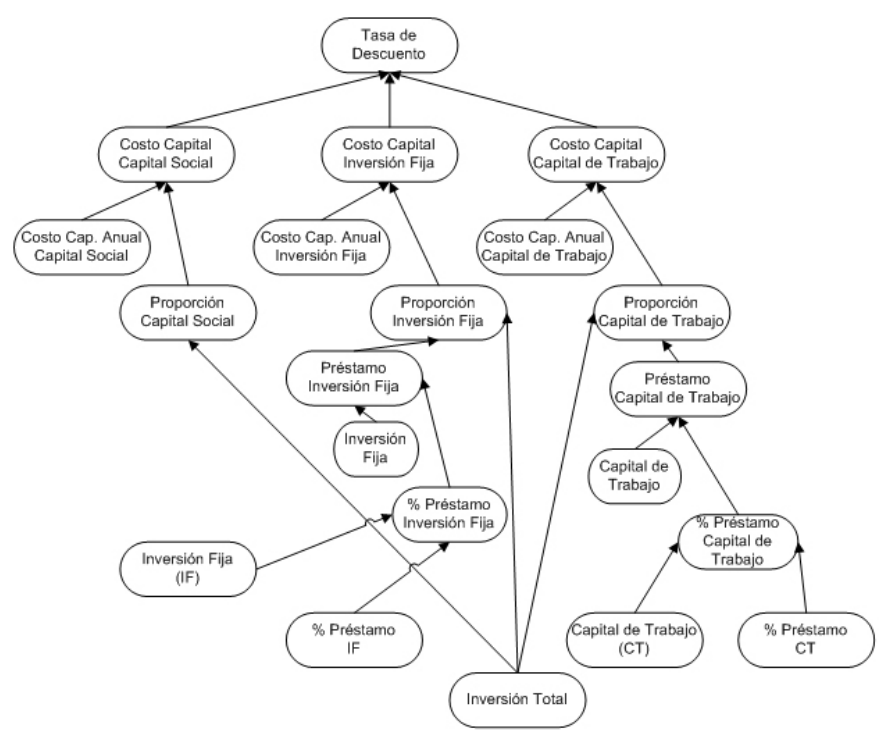

FIGURA 2.2. Diagrama de influencia para el cálculo de la tasa de descuento

El costo de la alimentación del personal (Cap) se muestra en (2.15), donde Tdm es el número de días por mes (21 días por mes), Tma es el número de meses por año (10 mes por año), CUap es el costo unitario de alimentación del personal por día (S/ 5.00 por persona) y $N e p_{j}$ es el número de envasadores que depende de la planta., donde $j=1, . ., 3$.

$$
C a p=T d m T m a C U a p\left(N p p_{j}+N e p_{j}\right) X_{j}
$$

Costos indirectos de fabricación (CIF) Los costos indirectos de fabricación se componen de los costos de mano de obra indirecta, suministro de agua, suministro de petróleo y suministro de energía eléctrica, tal como se observa en (2.16), donde CMOI es el costo de la mano de obra indirecta, CSa es el costo del suministro de agua, CSp es el costo del suministro de petróleo y CSee es el costo del suministro energía eléctrica.

$$
C I F=C M O I+C S a+C S p+C S e e
$$

El costo de mano de obra indirecta $(C M O I)$ se define en (2.17), donde $N j$ es el número de jefes $(N j=1$ para un jefe de planta) y $C U j$ es el costo unitario del jefe de planta en soles $(\mathrm{S} / 3,000)$.

$$
C M O I=N j(15 C U j(1+0,0975)+C U j)
$$

El costo de suministro de agua ( $C S a$ ) se define en (2.18), donde $K S a$ es el suministro de agua para el caldero en litros por caja (2.44 litros por caja) y CUSa es el costo unitario del suministro de agua en soles por metro cúbico (S/ 3.9590 por m3).

$$
C S a=K S a C U S a X_{j} Q_{t j}
$$

El costo del suministro de petróleo ( $C S p$ ) se define en (2.19), donde $K S p$ es el suministro de petróleo en galones por caja (depende de cada planta) y CUSp es el costo unitario del suministro de petróleo en soles por galón (S/ 11.2 por galón).

$$
C S p=K S p C U S p X_{j} Q_{t j}
$$

El costo de energía eléctrica ( $C S e$ ) se define en (2.20), donde $K S e e$ es el suministro de energía eléctrica en kilowatts por caja (depende de cada planta) y CUSee es el costo unitario del suministro de energía eléctrica en soles por kilowatts al mes (depende de cada planta).

$$
C S e e=K S e e C U S e e X_{j} Q_{t j}
$$

Depreciación del área de producción (DP) La depreciación del área de producción se compone de la del edificio, las instalaciones y las máquinas y equipos tal como se muestra en (2.21), donde De es la 
depreciación del edificio, $D i$ es la depreciación de las instalaciones y $D m e$ es la depreciación de las máquinas y equipos.

$$
D P=D e+D i+D m e
$$

La depreciación del edificio ( $D e$ ) se define en (2.22), donde $I N V e_{j}$ es la inversión del edificio (depende de cada planta), para $j=1, \ldots, 3$.

$$
D e=0,05 I N V e_{j} X_{j}
$$

La depreciación de las instalaciones (Di) se define en (2.23), donde $I i_{j}$ es la inversión en instalaciones (depende de cada planta), para $j=1, . ., 3$.

$$
D i=0,1 I i_{j} X_{j}
$$

La depreciación de las máquinas y equipos (Dme) se define en (2.24), La depreciación de las máquinas y equipos se define en (2.24), donde $I N V m e_{j}$ es la inversión en máquinas y equipos (depende de cada planta), para $j=1, \ldots, 3$.

$$
\text { Dme }=0,1 I_{N V m e} X_{j}
$$

2.2.3. Gastos operativos (GO) . Los gastos operativos se componen de gastos administrativos (GA) y gastos de ventas $(G V)$, tal como se observa en (2.25).

$$
G O=G A+G V
$$

Gastos administrativos (GA). Los gastos administrativos se definen en (2.26), donde $G g$ son los gastos de gerentes, $G s$ son los gastos en secretarias, $D a$ es la depreciación del área administrativa y $A$ es la amortización intangibles.

$$
G A=G g+G s+D A+A
$$

Los gastos de gerentes $(G g)$ se definen en (2.27), donde $N g$ es el número de gerentes y $S g$ es el sueldo del gerente en soles.

$$
G g=N g(15 S g(1+0,0975)+S g)
$$

Los gastos de secretarias $(G s)$ se definen en (2.28), donde $N s$ es el número de secretarias y $S s$ es el sueldo de las secretarias en soles.

$$
G s=N s(15 S s(1+0,0975)+S s)
$$

La depreciación del área administrativa (DA) se define en (2.29), donde $I N V e o_{j}$ es la inversión en equipos de oficina (depende de cada planta), para $j=1, . ., 3$ y la $I N V m_{j}$ es la inversión en muebles (depende de cada planta), para $j=1, \ldots, 3$.

$$
D A=\frac{1}{2}\left(0,25 I N V e o_{j}+0,1 I N V m_{j}\right) X_{j}
$$

La amortización de intangibles (A) se define en (2.30), donde INVint es la inversión en intangibles.

$$
A=0,2 I N \text { Vint }
$$

Gastos de ventas (GV). Los gastos de ventas se definen en (2.31), donde Gjv es el gasto del jefe de ventas, $G a$ es el gasto de asistentes, $G t f$ es el gasto total en ferias y $D V$ es la depreciación del área de ventas.

$$
G V=G j v+G a+G t f+D V
$$

Los gastos de jefe de ventas $(G j v)$ se definen en (2.32), donde $N j$ es el número de jefes $(N j=1$ para un jefe de ventas) y $C U j$ es el costo unitario del jefe de ventas en soles.

$$
G j v=N j(15 C U j(1+0,0975)+C U j)
$$


Los gastos de asistentes $(G a)$ se definen en (2.33), donde $N s$ es el número de secretarias y $S s$ es el sueldo de las secretarias en soles.

$$
G a=N s(15 S s(1+0,0975)+S s)
$$

Los gastos totales en ferias ( $G t f)$ se definen en (2.34), donde $N f$ es el número de ferias, en nuestro caso igual a uno y $G f$ es el gasto de participar en una feria.

$$
G t f=N f G f
$$

La depreciación del área de ventas $(D V)$ se define en (2.35), donde $I N V e o_{j}$ es la inversión en equipos de oficina (depende de cada planta), para $j=1, . ., 3$ y la $I N V m_{j}$ es la inversión en muebles (depende de cada planta), para $j=1, . ., 3$.

$$
D V=\frac{1}{2}\left(0,25 I N V e o_{j}+0,1 I N V m_{j}\right) X_{j}
$$

2.2.4. Cuotas del préstamo . La cuota está compuesta por el interés y la amortización de la deuda, ver (2.36) y la del año 1 se muestra en (2.37) y (2.38), en donde se considera periodo de gracia de la amortización de la deuda en los tres primeros trimestres del primer año, donde $I F_{j}$ es la inversión fija (depende de cada planta), para $j=1, . ., 3, C T_{j}$ es el capital de trabajo (depende de cada planta), para $j=$ $1, . ., 3$, PPif es el porcentaje de préstamo de la inversión fija, PPct es el porcentaje de préstamo del capital de trabajo, Tif es la tasa de interés trimestral del préstamo de la inversión fija y Tct es la tasa de interés trimestral del préstamo del capital de trabajo.

$$
\text { Cuota }_{t}=\text { Interés }_{t}+\text { Amortización }_{t}
$$

$$
\text { Cuota }_{1}=\text { Cuota } I F_{1}+\text { Cuota } C T_{1}
$$

$$
\begin{aligned}
\text { Cuota }_{1}= & {\left[3 \text { Tif }+\left(\frac{\text { Tif }(1+\text { Tif })^{17}}{(1+\text { Tif })^{17}-1}\right)\right]\left(\text { PPif IF }_{j} X_{j}\right) } \\
& +\left[3 T c t+\left(\frac{\text { Tct }(1+T c t)^{17}}{(1+T c t)^{17}-1}\right)\right]\left(\text { PPct } C T_{j} X_{j}\right)
\end{aligned}
$$

En (2.39) y (2.40) se muestran las conversiones de tasa de interés efectiva anual a tasa de interés trimestral de la inversión fija y del capital de trabajo, respectivamente, donde TIEAif es la tasa de interés efectiva anual del préstamo de la inversión fija y TIEAct es la tasa de interés efectiva anual del préstamo del capital de trabajo.

$$
\begin{aligned}
& \text { Tif }=\left((\text { TIEAif }+1)^{0,25}-1\right) \\
& \text { Tct }=\left((\text { TIEAct }+1)^{0,25}-1\right)
\end{aligned}
$$

La cuota del año 2 al año 5 es la misma, tal como se muestra en (2.41).

$$
\text { Cuota }_{t=2, \ldots, 5}=\left[4 \operatorname{PPifIF}\left(\frac{\text { Tif }(1+\text { Tif })^{17}}{(1+\text { Tif })^{17}-1}\right)+4 \operatorname{PPctCT}\left(\frac{\text { Tct }(1+\text { Tct })^{17}}{(1+\text { Tct })^{17}-1}\right)\right]
$$

2.2.5. Depreciación total (D) . La depreciación total está compuesta por la del área de producción $(D P)$, la del área de administración $(D A)$ y la del área de ventas $(D V)$ y se define en $(2.42)$.

$$
D=D P+D A+D V
$$

2.2.6. Impuesto a la renta (IR) . El impuesto a la renta se define en (2.43), donde $V$ son las ventas, $C P$ son los costos de producción, $G O$ son los gastos operativos y $G F$ son los gastos financieros.

$$
I R=0,3(V-C P-G O-G F)
$$


2.2.7. Gastos financieros (GF) . Los gastos financieros se definen en (2.44), donde $I_{t}$ es el interés en soles del año $t$, donde $t=1, . ., 5$.

$$
G F=I_{t}
$$

2.2.8. Costo de capital promedio ponderado financiero (CCPPf) . En (2.45) se muestra el costo de capital promedio ponderado que será considerado como tasa de descuento en la evaluación del proyecto, donde $X_{j}$ es la variable binaria, 1 si se construye la planta $j$ y 0 de otra manera, para $j=1,2$ y $3, I F_{j}$ es la inversión fija de la planta $j$ para $j=1, . ., 3, C T_{j}$ es el capital de trabajo de la planta $j$, para $j=1, . ., 3, P P i f$ es el porcentaje del préstamo en inversión fija, $P P c t$ es el porcentaje del préstamo en capital social y $C S$ es el capital social en soles.

$$
C C P P f=\frac{0,18 C S+0,14\left[\operatorname{PP} i f \sum_{j=1}^{3}\left(X_{j} I F_{j}\right)+\operatorname{PPct} \sum_{j=1}^{3}\left(X_{j} C T_{j}\right)\right]}{\sum_{j=1}^{3}\left[X_{j}\left(I F_{j}+C T_{j}\right)\right]}
$$

El coeficiente de 0.18 , es porque se asumió que el costo de oportunidad de capital del inversionista es de 18.00

Los coeficientes de 0.14 , es por la multiplicación de 0.20 y 0.70 . El coeficiente de 0.20 es porque se asumió que la tasa de interés efectiva anual tanto para el préstamo de la inversión fija, como para el capital de trabajo es de $20.00 \%$, y 0.70 por la diferencia entre $100 \%$ y $30 \%$ (impuesto a la renta).

\subsection{Formulación del modelo de programación no lineal (PNL) .}

2.3.1. Variables de decisión del modelo de PNL . Se consideró como variables de decisión para el modelo de programación no lineal las siguientes:

$X_{j}=1$ si se construye la planta $j$ y 0 de otra manera, donde $j=1, . ., 3 ;$ PPif $=$ porcentaje del préstamo en inversión fija; $P P c t=$ Porcentaje del préstamo en capital social y $C S=$ capital social en soles.

2.3.2. Función objetivo del modelo de PNL . La función objetivo para el siguiente modelo de PNL es la de maximizar el valor actual neto financiero, tal como se muestra en (2.46).

$$
\begin{aligned}
M A X Z= & 0,7 \sum_{t=1}^{5} \frac{\sum_{j=1}^{3}(V-C M D-C M O D-C S a-C S p-C S e e-D P-D A-D V)}{(1+C C P P f)^{t}} \\
& -0,7 \sum_{t=1}^{5} \frac{C M O I+G g+G s+A+G j v+G a+G f}{(1+C C P P f)^{t}} \\
& -\sum_{t=1}^{5} \frac{C U O T A_{t}-D-A-0,3 G F}{(1+C C P P f)^{t}}-\sum_{j=1}^{3}\left(I F_{j} X_{j}+C T_{j} X_{j}\right)
\end{aligned}
$$

El coeficiente de 0.7 en la función objetivo de maximización resultó de restar $100 \%$ y el impuesto a la renta que es igual a $30 \%$.

2.3.3. Restricciones del modelo de PNL . Se consideró las siguientes restricciones: Para implementar una sola planta se define en (2.47).

$$
\sum_{j=1}^{3} X_{j}=1
$$

La producción máxima de cajas por disponibilidad de materia prima se define en (2.48).

$$
\sum_{j=1}^{3} X_{j} Q_{j} \leq 300,000
$$

La disponibilidad de capital social se define en (2.49).:

$$
C S \leq 1,000,000
$$


La inversión total se define en (2.50).

$$
C S+P \operatorname{Pif} \sum_{j=1}^{3}\left(X_{j} I F_{j}\right)+P P c t \sum_{j=1}^{3}\left(X_{j} C T_{j}\right) \leq \sum_{j=1}^{3} X_{j}\left(I F_{j}+C T_{j}\right)+0,00001
$$

El coeficiente de 0.00001 sirve para no hacer indeterminado la solución del costo de capital promedio ponderado y con ello tener una solución factible.

El préstamo máximo se define en (2.51).

$$
\text { PPif }+ \text { PPct } \leq 0,6 \sum_{j=1}^{3}\left[X_{j}\left(I F_{j}+C T_{j}\right)\right]
$$

2.4. Simulación Monte Carlo del proyecto. En la simulación Monte Carlo del proyecto se consideraron las siguientes variables aleatorias.

2.4.1. Variable aleatoria de abastecimiento de materia prima. La disponibilidad de anchoveta es una variable aleatoria a tomar en cuenta porque depende de las cuotas de pesca, temporadas de pesca, estación del año y fenómenos climatológicos como el Fenómeno El Niño, haciéndolo imprevisible para hacer programas de producción.

Con registros de desembarque de anchoveta destinadas a conservas entre enero de 2008 a diciembre de 2011, se obtuvo con el programa StatFit de Promodel, que los desembarques siguen una distribución Lognormal $(-7.18 \mathrm{e}+003,9.53,0.28)$. En las Figuras 2.3 y 2.4 se observan la función de densidad y la función de distribución del desembarque de anchoveta destinada a conservas.

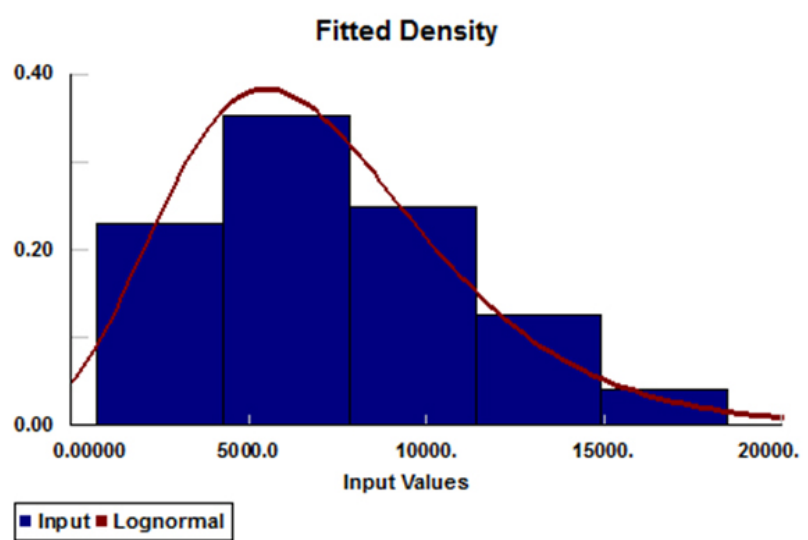

FIGURA 2.3. Función de densidad de probabilidad de la disponibilidad de anchoveta para conserva

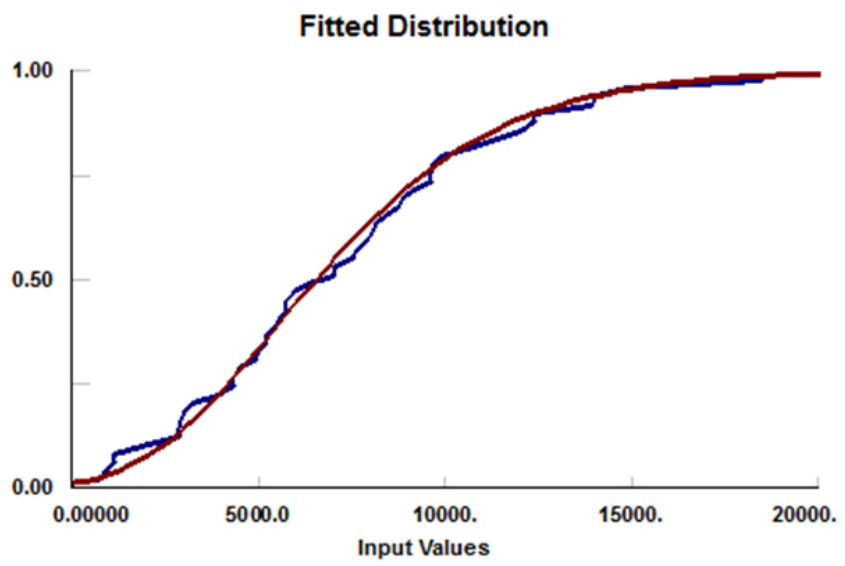

FIGURA 2.4. Función de distribución de probabilidad de la disponibilidad de anchoveta para conserva 
2.4.2. Variables aleatorias en la planta. Las principales variables aleatorias en la planta son el rendimiento de la materia prima, que está en función de la calidad de la materia prima, y la eficiencia del caldero. Dentro de la experiencia práctica en planta, se consideró una distribución de probabilidad normal de media $81.18 \%$ y desviación estándar $1 \%$ para el rendimiento de la materia prima, y media $75 \%$ y desviación estándar de $3 \%$ para la eficiencia del caldero.

2.4.3. Variables aleatorias de mercado. Dentro de las variables aleatorias de mercado se consideraron la demanda cuantitativa de conservas, precio de las conservas y el precio del petróleo.

En el Cuadro 2.8 se muestra la demanda anual en caja de conservas de tres mercados simulados.

CUADro 2.8

Demanda anual (en cajas de conservas)

\begin{tabular}{crrr}
\hline Año & Mercado 1 & Mercado 2 & Mercado 3 \\
\hline 1 & 80,000 & 100,000 & 120,000 \\
2 & 93,000 & 105,000 & 130,000 \\
3 & 85,000 & 115,000 & 122,000 \\
4 & 111,000 & 120,000 & 140,000 \\
5 & 120,000 & 130,000 & 155,000 \\
\hline
\end{tabular}

Para determinar la función de densidad de la demanda del mercado se utilizó el programa StatFit de Promodel. Con este programa se determinó que sigue una distribución normal de media igual a 115,067 cajas y desviación estándar igual a 20,264 cajas. En las Figuras 2.5 y 2.6 se observan la función de densidad y la función de distribución de la demanda del mercado.

Fitted Density

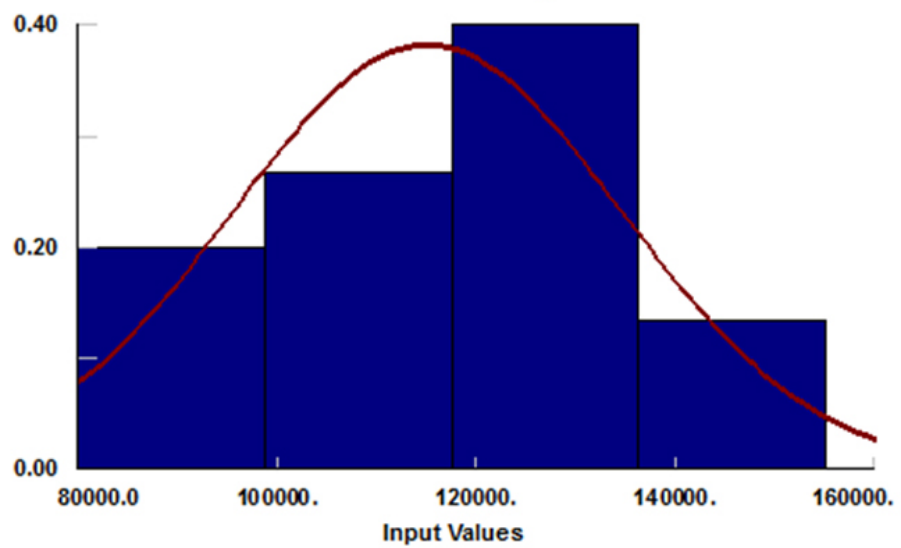

FIGURA 2.5. Función de densidad de probabilidad de la demanda de conservas

\section{Fitted Distribution}

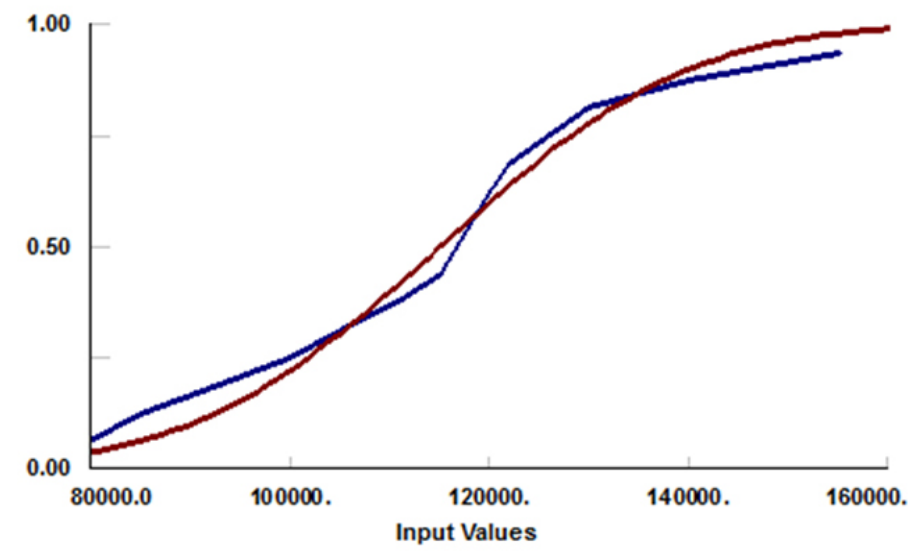

FIGURA 2.6. Función de distribución de probabilidad de la demanda de conservas 
En la determinación de la función de densidad del precio de las conservas se utilizó el mismo programa que para la demanda de conservas, el StatFit de Promodel y se encontró de que sigue una distribución normal de media igual a S/ 2.9 y desviación estándar igual a S/ 3.38e-002. En las Figuras 2.7 y 2.8 se muestran la función de densidad de probabilidad y la función de distribución de probabilidad del precio de la lata de conserva de anchoveta respectivamente.

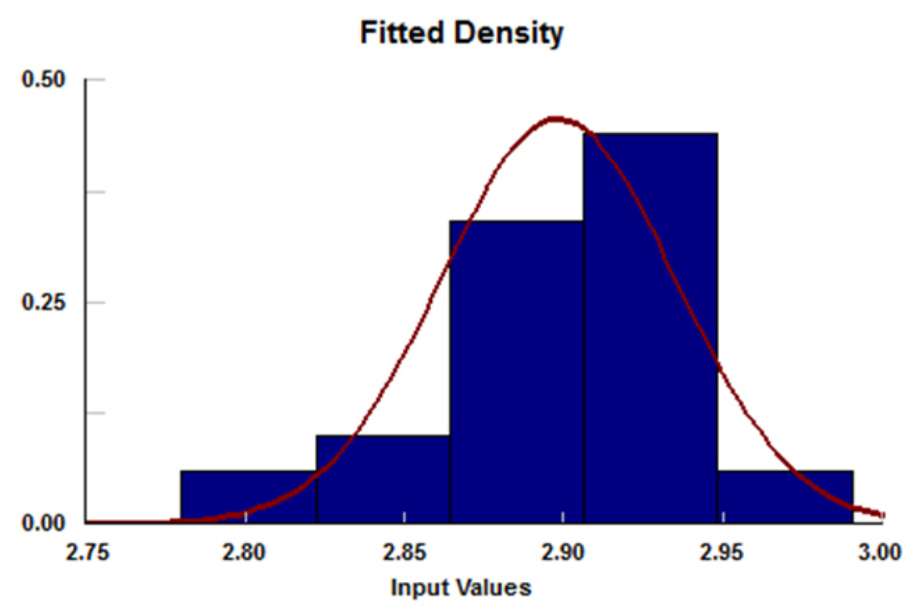

FIGURA 2.7. Función de densidad de probabilidad del precio de la lata de conserva de anchoveta

\section{Fitted Distribution}

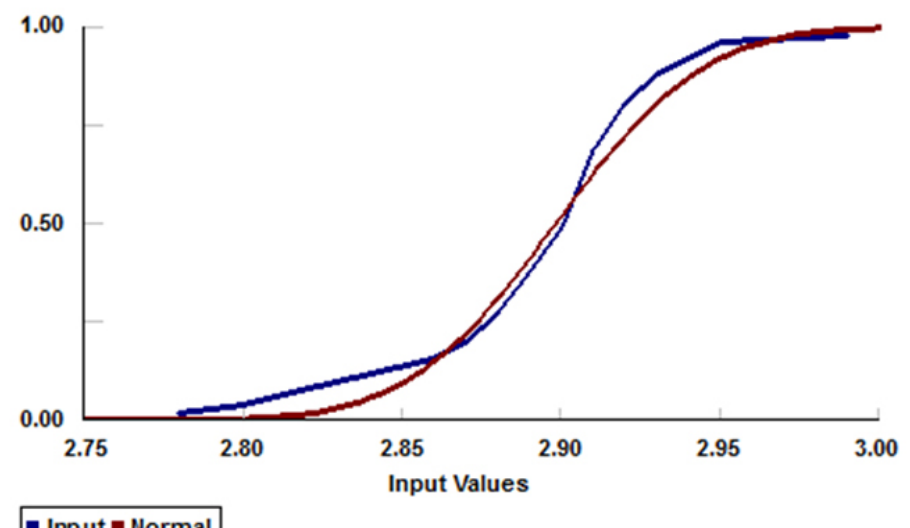

- Input $\mathbf{n}$ Normal

FIGURA 2.8. Función de distribución de probabilidad del precio de la lata de conserva de anchoveta

Se utilizaron datos del precio del petróleo de enero de 2006 a mayo de 2012 y con el StatFit de Promodel se obtuvo que el precio del petróleo sigue una distribución normal de media igual a S/ 9.53 y desviación estándar igual a S/ 0.734. En las Figuras 2.9 y 2.10 se muestran la función de densidad de probabilidad y la función de distribución de probabilidad del precio del petróleo Diesel 2.

\section{Resultados y discusiones.}

3.1. Modelo de programación no lineal. La solución del Solver de Excel recomienda que para optimizar la función objetivo de maximizar el valor actual neto financiero (VANF) se debe instalar la planta 3, con un porcentaje de préstamo del $81.01 \%$ y $15.29 \%$ para la inversión fija y capital de trabajo respectivamente, y utilizar S/ 876,000 de S/ 1,000,000 que se tenía como capital social.

El valor actual neto financiero al $19.20 \%$ de tasa de costo de capital promedio ponderado es de S/ $14,503,152$ y S/ 15,321,782 sin liquidación y con liquidación respectivamente, y para la tasa interna de retorno financiero (TIRF) es de $516.80 \%$ sin liquidación y $516.91 \%$ con liquidación.

3.2. Simulación Monte Carlo. Se utilizó para hacer la simulación Monte Carlo el programa @Risk con una corrida con 1,000 iteraciones.

En los resultados de las corridas de simulación se consideró como variables de salida el VANF sin liquidación y con liquidación, y el TIRF sin liquidación y con liquidación. 


\section{Fitted Density}

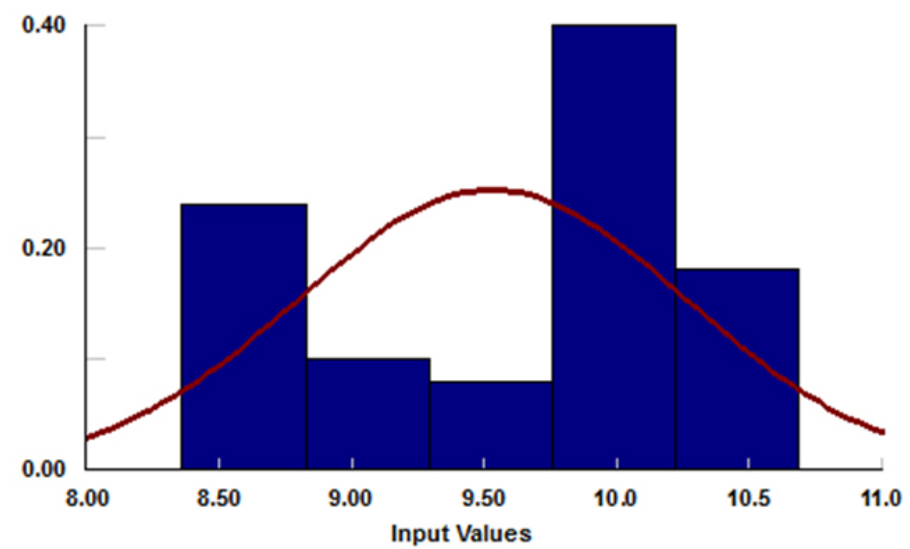

FIgURA 2.9. Función de densidad de probabilidad del precio del Petróleo Diésel 2

\section{Fitted Distribution}

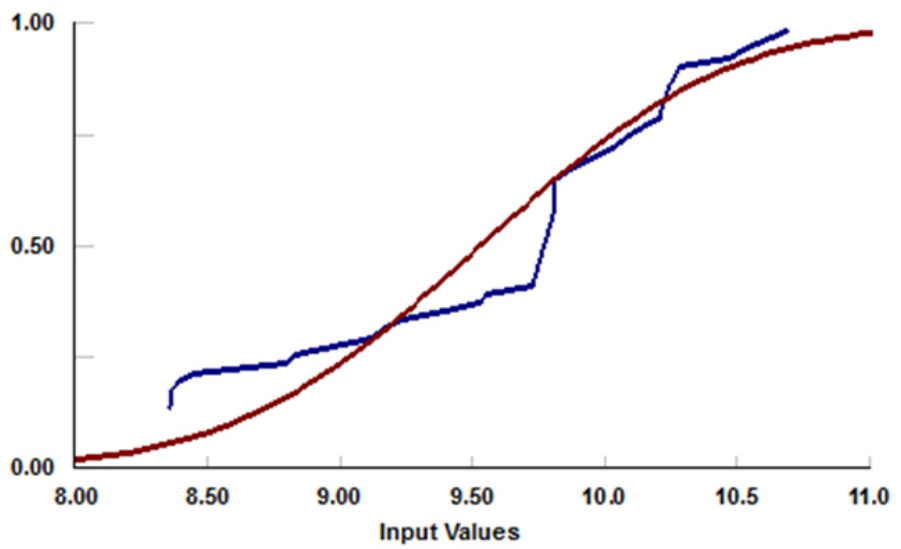

FIGURA 2.10. Función de distribución de probabilidad del precio del Petróleo Diésel 2

Al hacer la simulación Monte Carlo se obtuvo para el VANF sin liquidación un mínimo de S/ 10,290,572, un máximo de S/ 22,102,304 y una media de S/ 16,517,086; para el VANF con liquidación un mínimo de S/ $11,109,202$, un máximo de S/ 22,920,935 y una media de S/ 17,335,717 tal como se observa en las Figuras 3.1 y 3.2 .

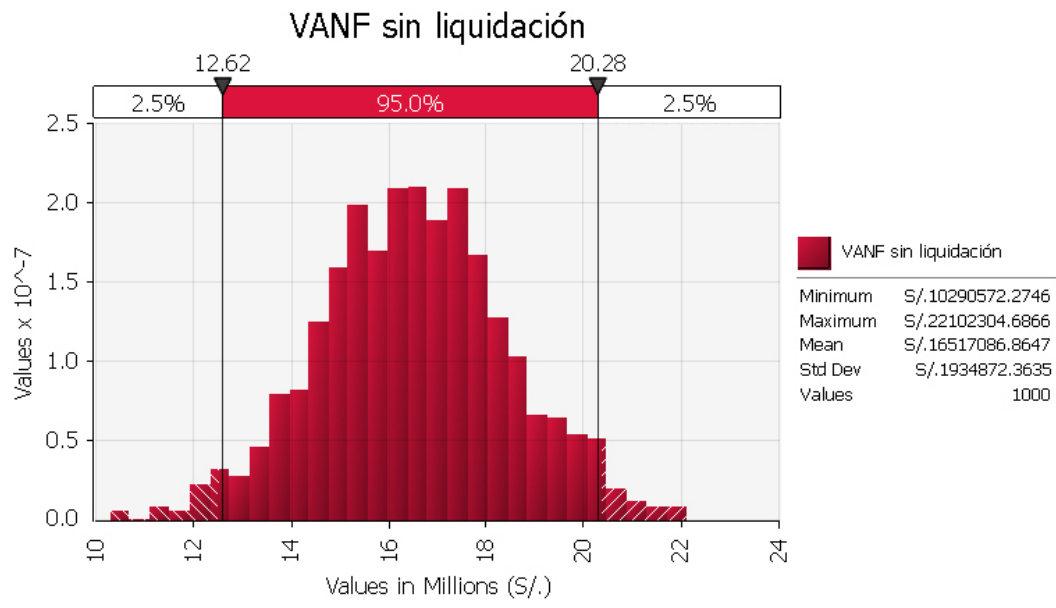

FIGURA 3.1. Función de Densidad de Probabilidad del VANF sin liquidación 


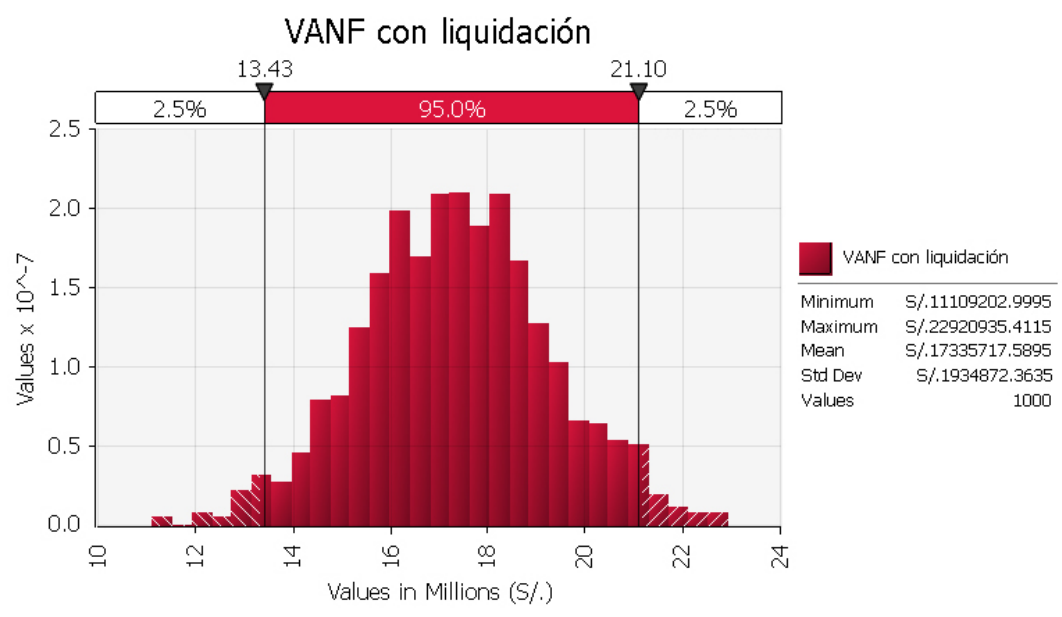

FIGURA 3.2. Función de Densidad de Probabilidad del VANF con liquidación

Se obtuvo para el TIRF sin liquidación un mínimo de $277 \%$ un máximo de $948 \%$ y una media de $587 \%$; para el TIRF con liquidación un mínimo de $277 \%$, un máximo de $948 \%$ y una media de $587 \%$ tal como se observa en las Figuras 3.3 y 3.4 .

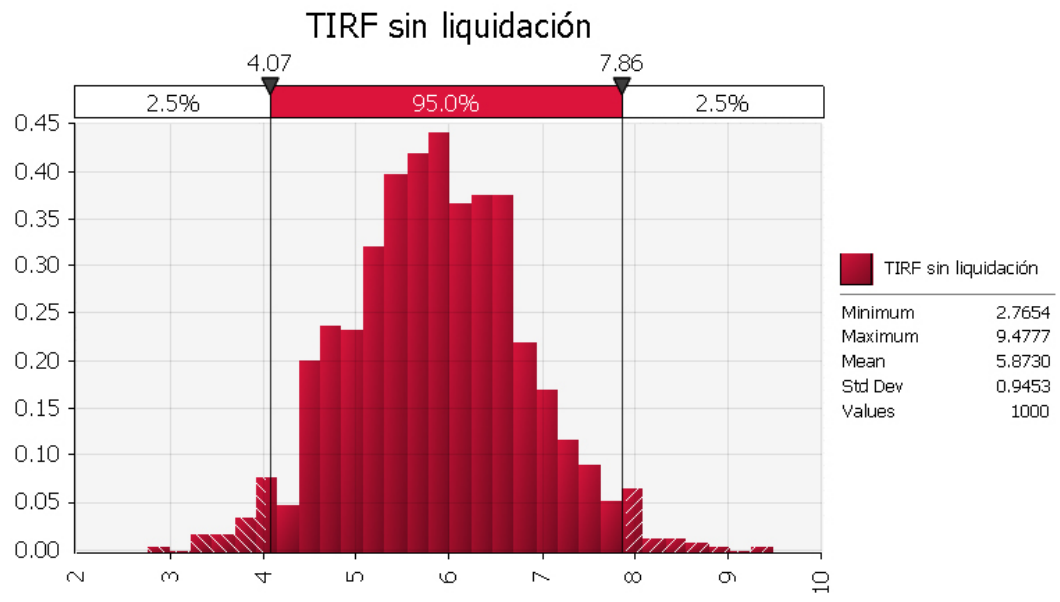

FIGURA 3.3. Función de Densidad de Probabilidad del TIRF sin liquidación

En la gráfica acumulada ascendente de la Figura 3.5 se observa que el VANF con liquidación tiene una menor probabilidad $(0.6 \%)$ de tener un valor menor a S/ 12,620,000, sin embargo el VANF sin liquidación tiene una mayor probabilidad $(95.0 \%$ ) de que se obtenga un valor entre S/ 12,620,000 y S/ 20,280,000.

Según los resultados de la simulación se puede observar que hay un $95 \%$ de confianza de que se puede obtener un VANF sin liquidación entre S/ 12,620,000 y S/ 20,280,000; y entre S/ 13,430,000 y S/ 21,100,000 para el VANF con liquidación tal como se muestra en las Figuras 3.1 y 3.2. En el caso de la evaluación del TIRF sin liquidación, se puede decir que existe un $95 \%$ de confianza de que está entre $407 \%$ y $786 \%$; y entre $408 \%$ y $786 \%$ para el TIRF con liquidación, tal como se observa en las Figuras 3.3 y 3.4.

Como se puede observar en la metodología lo primero que se realizó fue optimizar y luego simular, pero que hubiera pasado si se hubiera simulado primero y luego optimizado. Para responder a esa inquietud se tomará el concepto de teoría de decisiones, allí se considera que existen alternativas de decisión y eventos o estados de la naturaleza. Lo primero que se hace es tomar la decisión y luego suceden los eventos con lo que se obtienen resultados estimados, en cambio sí primero suceden los eventos se podría tomar una mejor decisión en base a lo ocurrido, pero como se trata de eventos aleatorios podría cambiar después de la decisión tomada, con lo cual sería difícil determinar si es mejor o no hacer primero la optimización que la simulación. 


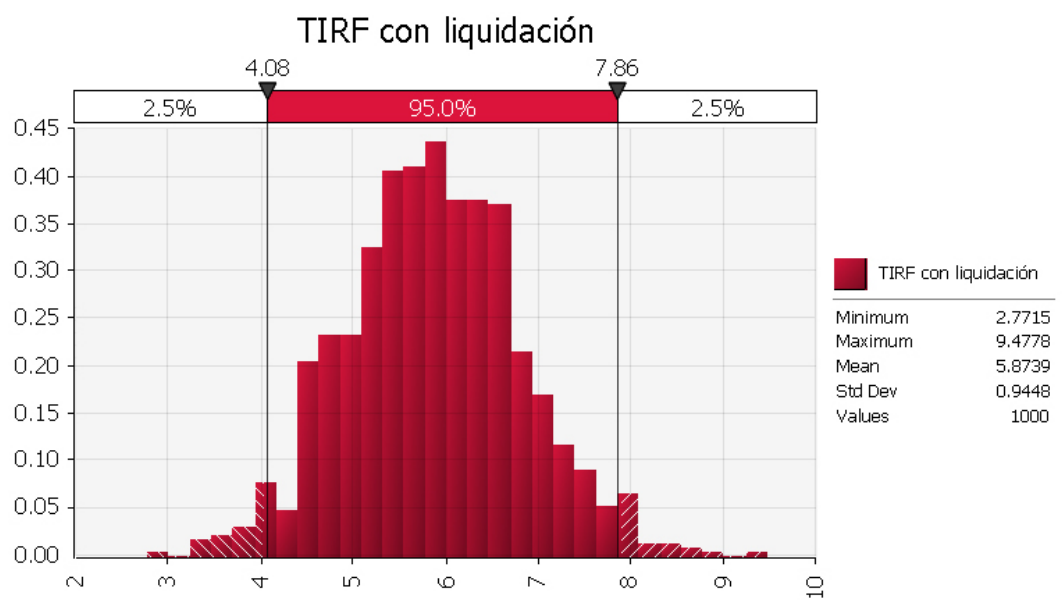

FIGURA 3.4. Función de Densidad de Probabilidad del TIRF con liquidación

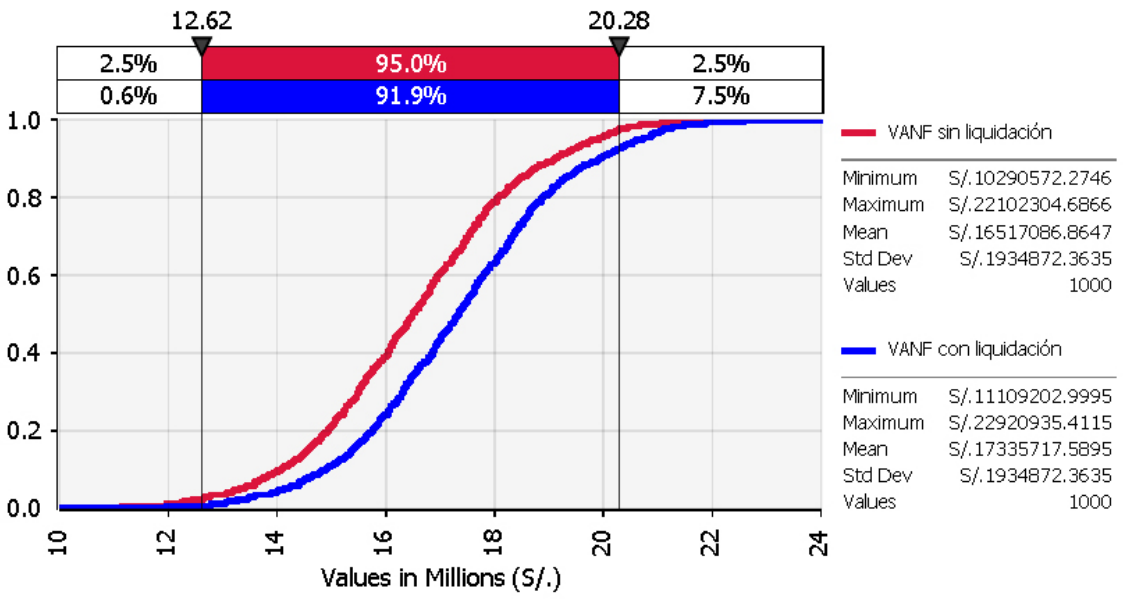

FIGURA 3.5. Gráfica acumulada ascendente del VANF sin liquidación y con liquidación

\section{Conclusiones.}

1. En la construcción del modelo matemático de un proyecto industrial, se pueden plantear varias funciones objetivos, convirtiéndose en un modelo de programación multiobjetivo en donde lo que se busca no es la optimización sino la solución más eficiente. En este trabajo de investigación solo se maximizó el valor actual neto financiero para determinar que planta instalar, además de los porcentajes de los préstamos para la inversión fija, capital de trabajo y el capital social, pero podría optimizarse otras funciones como minimizar costos o tiempos de finalización de proyectos [18].

2. En los proyectos industrial pesqueros se realizan cálculos de ingeniería para determinar el balance de materia y energía, el número y capacidades de las máquinas, así como el espacio requerido para la instalación. Es importante el nivel de elaboración del proyecto, porque a nivel de perfil, a no requerir mayores cálculos de ingeniería podría tener mayor riesgo tomar una decisión que en un proyecto a nivel de prefactibilidad donde se hacen cálculos estimados en ingeniería.

5. Agradecimientos. Un agradecimiento especial a todas las personas que colaboraron directamente o indirectamente con la realización de este trabajo de investigación.

ORCID and License

Christian Ramos Angeles https://orcid.org/0000-0002-2275-2858,

Pedro Espinoza Haro https: / / orcid.org/0000-0002-8018-1790.

This work is licensed under the Creative Commons Attribution-NoComercial-ShareAlike 4.0.

[1] Aboelmagd Y.M.R. Linear programming applications in construction sites. Alexandria Engineering Journal. 2018; 57: 4177 4187. 
[2] Armaneri Ö., Özdağoğlu G., Yalçinkaya Ö. An integrated decision support approach for project investors in risky and uncertain environments. Journal of Computational and Applied Mathematics. 2010; 234:2530-2542.

[3] Bhattacharyya R., Kumarb P., Kar S. Fuzzy R\&D portfolio selection of interdependent projects. Computers and Mathematics with Applications. 2011; 62:3857-3870.

[4] Chevalier E., Vath V.L., Roch A., Scotti S. Optimal exit strategies for investment projects. Journal of Mathematical Analisys and Applications. 2015; 425:666-694.

[5] De Souza J.S., Neto F.J., Anzanello M.J., Filomena T.P. A non-traditional capital investment criteria-based method to optimize a portfolio of investments. International Journal of Industrial Engineering. 2010; 19(4):193-203.

[6] Ji X., Zhu S., Wang S., Zhang S. A stochastic linear goal programming approach to multistage portfolio management based on scenario generation via linear programming. IIE Transactions. 2005; 37:957-969.

[7] Klanšek U., Pšunder M. Cost Optimal Project Scheduling. Organizacija. 2008; 41(4):153-8.

[8] Kelliher Ch.F., Mahoney L.S. Using Monte Carlo Simulation to improve long-term investment decisions. The Appraisal Journal. 2000; 68(1):44-56.

[9] Lohmann, J., Baksh, S. The IRR, NPV and Payback Period and their relative performance in common capital budgeting decision procedures for dealing with risk. The Engineering Economist. 1994; 39(1):17-47.

[10] Manotas D.F. Optimal economic project selection under uncertainty: an illustration from an utility company. Ingeniería y Competitividad. 2009; 11(2)41-52.

[11] Milanovic D.Lj., Milanovic D.D., Misita, M. The evaluation of risky investment projects. FME Transactions. $2010 ; 38$ : 103-6.

[12] Moussourakis J., Haksever C. Project Compression with Nonlinear Cost Functions. Journal of Construction Engineering and Management. 2010; 136(2):251-9.

[13] Nawrocki, D., The problems with Monte Carlo simulation. Journal of Financial Planning. 2001; 14(11):92-106.

[14] Platon V., Constantinescu A. Monte Carlo Method in risk analysis for investment projects. Procedia Economics and Finance. 2014; 15:393-400.

[15] Nguyen T.T. Portfolio selection under higher moments using fuzzy multi-objective linear programming. Journal of Intelligent \& Fuzzy Systems. 2016; 30:2139-2156.

[16] Padberg M., Wilczak M.J. Optimal Project Selection When Borrowing and Lending Rates Differ. Mathematical and Computer Modelling. 1999; 29:63-78.

[17] Poveda G. Modelo matemático y dimensional para el planeamiento óptimo de industrias de procesos, $1^{\circ} \mathrm{ed}$. Medellín, Colombia: Instituto Tecnológico Metropolitano; 2008.

[18] Ramos C.R., Flores C.A. Reducción del tiempo de finalización del proyecto de una planta de conservas de pescado utilizando un modelo de programación lineal. Anales Científicos UNALM. 2016; 77(1):110-7.

[19] Taylor B.W., Moore L.J., Clayton E.R. R\&D project selection and manpower allocation with integer nonlinear goal programming. Management Science. 1982; 28(10): 1149-1158.

[20] Zhang X.-S., Cui J.-C. A project evaluation system in the state economic information system of China: An operations research practice in public sectors. International Transactions in Operational Research. 1999; 6:441-452.

[21] Rudenko Z.G. Nonlinear Optimization Problem of Interdependent Investment Projects Portfolio. Automation and Remote Control. 2016; 77(10):1849-1854. 